\title{
Comparison of two bounds of the quantum correlation set
}

\author{
David Avis \\ School of Computer Science \\ McGill University \\ 3480 University, Montreal, Quebec, Canada H3A 2A7 \\ Email: avis@cs.mcgill.ca
}

\author{
Tsuyoshi Ito \\ Department of Computer Science \\ The University of Tokyo \\ 7-3-1 Hongo, Bunkyo-ku, Tokyo, 113-0033 Japan \\ Email: tsuyoshi@is.s.u-tokyo.ac.jp
}

\begin{abstract}
From a geometric viewpoint, quantum nonlocality between two parties is represented as the difference of two convex bodies, namely the sets of possible results of classical and quantum correlation experiments, the latter of which is called the quantum correlation set. Whereas little is known about the quantum correlation set, Tsirelson's theorem (1980) can be seen as the exact characterization of possible pairwise quantum correlations, where mean values of individual observables are discarded. In this paper, we compare two previously shown bounds of the quantum correlation set in the case where two parties have $m$ and $n$ choices of dichotomic observables, respectively. One bound comes from the direct application of Tsirelson's theorem and the no-signalling condition. The other bound, recently introduced by Avis, Imai and Ito, refines the application of Tsirelson's theorem in the previous bound. We show that for any $m, n \geq 2$, this new bound is strictly tighter than the earlier bound. In other words, there are correlations that satisfy Tsirelson's theorem, but are not realizable in a quantum setting.
\end{abstract}

\section{Problem And Result}

We consider (quantum) correlation experiments (see e.g. [1], [2]) by two parties Alice and Bob, where Alice has $m$ choices of \pm 1 -valued observables $A_{1}, \ldots, A_{m}$ and Bob has $n$ choices $B_{1}, \ldots, B_{n}$. Suppose that we are given $m n$ real numbers $x_{\mathrm{A}_{i} \mathrm{~B}_{j}}(1 \leq i \leq m, 1 \leq j \leq n)$. Tsirelson's theorem [3] (see [4] for proof) gives a beautiful exact condition that there exists a correlation experiment which satisfies $\left\langle A_{i} B_{j}\right\rangle=x_{\mathrm{A}_{i} \mathrm{~B}_{j}}$, where $\left\langle A_{i} B_{j}\right\rangle$ denotes the expected value of the product of the observables $A_{i}$ and $B_{j}$ : such a correlation experiment exists if and only if there exist $m+n$ unit vectors $\boldsymbol{u}_{i}, \boldsymbol{v}_{j} \in \mathbb{R}^{m+n}$ such that $x_{\mathrm{A}_{i} \mathrm{~B}_{j}}=\boldsymbol{u}_{i} \cdot \boldsymbol{v}_{j}$.

In the study of combinatorial optimization, the set of $m n$ dimensional vectors $\boldsymbol{x}$ satisfying this condition is referred to as the elliptope $\mathcal{E}\left(\mathrm{K}_{m, n}\right)$ of the complete bipartite graph $\mathrm{K}_{m, n}=\left(V_{m, n}, E_{m, n}\right)$ (see e.g. [5]). The $m+n$ vertices of this graph correspond to the $m+n$ observables partitioned in the obvious way into Alice's and Bob's observables. The $m n$ edges correspond to the $m n$ possible correlations between them. Generally, the elliptope of a graph $G=(V, E)$ is the set of $|E|$-dimensional vectors $\boldsymbol{x}$ which can be represented as $x_{i j}=\boldsymbol{u}_{i} \cdot \boldsymbol{u}_{j}$ by using $|V|$ unit vectors $\boldsymbol{u}_{i} \in \mathbb{R}^{|V|}$, each of which is associated with each vertex $i$ of $G$.

Next suppose we are additionally given $m+n$ real numbers $x_{\mathrm{XA}_{i}}(1 \leq i \leq m)$ and $x_{\mathrm{XB}_{j}}(1 \leq j \leq n)$, and we would like to tell whether there exists a quantum correlation experiment which satisfies $\left\langle A_{i}\right\rangle=x_{\mathrm{XA}_{i}},\left\langle B_{j}\right\rangle=x_{\mathrm{XB}_{j}}$ as well as $\left\langle A_{i} B_{j}\right\rangle=x_{\mathrm{A}_{i} \mathrm{~B}_{j}}$. We consider $\boldsymbol{x}$ as an $(m+n+m n)$ dimensional vector and denote the set of the vectors $\boldsymbol{x}$ which satisfies this condition by $\mathcal{Q}_{\text {Cut }}(m, n)$, called the quantum correlation set, following [6]. Here the appropriate graph is $\nabla \mathrm{K}_{m, n}=\left(\nabla V_{m, n}, \nabla E_{m, n}\right)$ which is the suspension graph of $\mathrm{K}_{m, n}$, that is obtained from $\mathrm{K}_{m, n}$ by adding a new vertex $\mathrm{X}$ and $m+n$ edges $\mathrm{XA}_{i}$ and $\mathrm{XB}_{j}$. These additional edges correspond to the mean values of the $m+n$ observables. Two bounds of $\mathcal{Q}_{\mathrm{Cut}}(m, n)$ can be derived easily. First, if we ignore $x_{\mathrm{XA}_{i}}$ and $x_{\mathrm{XB}_{j}}$ and only look at $x_{\mathrm{A}_{i} \mathrm{~B}_{j}}$, then we can use Tsirelson's theorem. This implies that $\pi\left(\mathcal{Q}_{\mathrm{Cut}}(m, n)\right)=$ $\mathcal{E}\left(\mathrm{K}_{m, n}\right)$ and therefore $\mathcal{Q}_{\text {Cut }}(m, n) \subseteq \pi^{-1}\left(\mathcal{E}\left(\mathrm{K}_{m, n}\right)\right)$, where $\pi: \mathbb{R}^{\nabla E_{m, n}} \rightarrow \mathbb{R}^{E_{m, n}}$ is the standard projection. Next, in correlation experiments, the joint probability that $A_{i}$ measures to $a \in\{ \pm 1\}$ and $B_{j}$ measures to $b \in\{ \pm 1\}$ is equal to $\left(1+a\left\langle A_{i}\right\rangle+b\left\langle B_{j}\right\rangle+a b\left\langle A_{i} B_{j}\right\rangle\right) / 4$. Therefore, if $\boldsymbol{x} \in$ $\mathcal{Q}_{\mathrm{Cut}}(m, n)$, then $1+a x_{\mathrm{XA}_{i}}+b x_{\mathrm{XB}_{j}}+a b x_{\mathrm{A}_{i} \mathrm{~B}_{j}} \geq 0$ for all $i, j$ and $a, b \in\{ \pm 1\}$. This is equivalent to consider the correlations satisfying the nonnegativity, normalization, and no-signalling conditions, see [1], [6]. ${ }^{1}$ The set of vectors $\boldsymbol{x}$ satisfying these $4 m n$ linear inequalities is referred to as the rooted semimetric polytope $\operatorname{RMet}\left(\nabla \mathrm{K}_{m, n}\right)$ (again see $\left.[5]^{2}\right)$. Therefore, we have $\mathcal{Q}_{\text {Cut }}(m, n) \subseteq \operatorname{RMet}\left(\nabla \mathrm{K}_{m, n}\right)$. Combining these two bounds, we have

$$
\mathcal{Q}_{\mathrm{Cut}}(m, n) \subseteq \pi^{-1}\left(\mathcal{E}\left(\mathrm{K}_{m, n}\right)\right) \cap \operatorname{RMet}\left(\nabla \mathrm{K}_{m, n}\right) .
$$

In [6], Avis, Imai and Ito proved that

$$
\mathcal{Q}_{\mathrm{Cut}}(m, n) \subseteq \mathcal{E}\left(\nabla \mathrm{K}_{m, n}\right) \cap \operatorname{RMet}\left(\nabla \mathrm{K}_{m, n}\right)
$$

as an application of Tsirelson's theorem. Since

$$
\begin{aligned}
\mathcal{E}\left(\nabla \mathrm{K}_{m, n}\right) \cap \operatorname{RMet} & \left(\nabla \mathrm{K}_{m, n}\right) \\
& \subseteq \pi^{-1}\left(\mathcal{E}\left(\mathrm{K}_{m, n}\right)\right) \cap \operatorname{RMet}\left(\nabla \mathrm{K}_{m, n}\right),
\end{aligned}
$$

the bound (2) is not worse than the bound (1). But it remained open whether the inclusion (3) is proper or not. In this paper, we answer this question affirmatively:

\footnotetext{
${ }^{1}$ The inequalities directly correspond to the nonnegativity condition. The normalization and no-signalling conditions are used to derive the fact that the joint probability is equal to $\left(1+a\left\langle A_{i}\right\rangle+b\left\langle B_{j}\right\rangle+a b\left\langle A_{i} B_{j}\right\rangle\right) / 4$.

${ }^{2}$ This is not the definition of the rooted semimetric polytope, but it is equivalent as proved in [6].
} 
Theorem 1: The inclusion (3) is proper for $m, n \geq 2$.

A corollary follows from Theorem 1 and (2).

Corollary 1: The inclusion (1) is proper for $m, n \geq 2$.

Corollary 1 implies that looking at the values $x_{\mathrm{A}_{i} \mathrm{~B}_{j}}$ of correlation functions is not enough to test whether a given vector $\boldsymbol{x}$ is realizable in a quantum correlation experiment, even if $\boldsymbol{x}$ is guaranteed to be no-signalling.

\section{Proof of Theorem 1}

First we prove the case of $(m, n)=(2,2)$.

The vector $\boldsymbol{x} \in \mathbb{R}^{\nabla E_{2,2}}$ defined by

$$
\begin{gathered}
x_{\mathrm{XA}_{1}}=x_{\mathrm{XA}_{2}}=0, x_{\mathrm{XB}_{1}}=x_{\mathrm{XB}_{2}}=1-1 / \sqrt{2}, \\
x_{\mathrm{A}_{1} \mathrm{~B}_{1}}=x_{\mathrm{A}_{1} \mathrm{~B}_{2}}=x_{\mathrm{A}_{2} \mathrm{~B}_{1}}=1 / \sqrt{2}, x_{\mathrm{A}_{2} \mathrm{~B}_{2}}=-1 / \sqrt{2}
\end{gathered}
$$

lies in $\pi^{-1}\left(\mathcal{E}\left(\mathrm{K}_{2,2}\right)\right) \cap \operatorname{RMet}\left(\nabla \mathrm{K}_{2,2}\right)$. The membership to $\operatorname{RMet}\left(\nabla \mathrm{K}_{2,2}\right)$ can be verified by straightforward calculation. The membership to $\pi^{-1}\left(\mathcal{E}\left(\mathrm{K}_{2,2}\right)\right)$ is proved by the fact that the coordinates of $\pi(\boldsymbol{x})$ are the inner products of the following unit vectors in $\mathbb{R}^{2}$ :

$$
\boldsymbol{u}_{1}=\left(\begin{array}{l}
1 \\
0
\end{array}\right), \boldsymbol{u}_{2}=\left(\begin{array}{l}
0 \\
1
\end{array}\right), \boldsymbol{v}_{1}=\frac{1}{\sqrt{2}}\left(\begin{array}{l}
1 \\
1
\end{array}\right), \boldsymbol{v}_{2}=\frac{1}{\sqrt{2}}\left(\begin{array}{c}
1 \\
-1
\end{array}\right) .
$$

We prove that the vector $\boldsymbol{x}$ does not belong to $\mathcal{E}\left(\nabla \mathrm{K}_{2,2}\right)$. Suppose the opposite. Then there exists unit vectors $\boldsymbol{u}_{1}^{\prime}, \boldsymbol{u}_{2}^{\prime}, \boldsymbol{v}_{1}^{\prime}, \boldsymbol{v}_{2}^{\prime}, \boldsymbol{w}^{\prime}$ which correspond to $\mathrm{A}_{1}, \mathrm{~A}_{2}, \mathrm{~B}_{1}, \mathrm{~B}_{2}, \mathrm{X}$, respectively, whose inner products give the coordinates of $\boldsymbol{x}$. However,

$$
\begin{aligned}
0 \leq & \left|\boldsymbol{u}_{1}^{\prime}-(1 / \sqrt{2})\left(\boldsymbol{v}_{1}^{\prime}+\boldsymbol{v}_{2}^{\prime}\right)+(\sqrt{2}-1) \boldsymbol{w}^{\prime}\right|^{2} \\
& +\left|2(\sqrt{2}-1) \boldsymbol{u}_{2}^{\prime}-(1 / \sqrt{2})\left(\boldsymbol{v}_{1}^{\prime}-\boldsymbol{v}_{2}^{\prime}\right)\right|^{2} \\
= & \left|\boldsymbol{u}_{1}^{\prime}\right|^{2}+4(3-2 \sqrt{2})\left|\boldsymbol{u}_{2}^{\prime}\right|^{2}+\left|\boldsymbol{v}_{1}^{\prime}\right|^{2}+\left|\boldsymbol{v}_{2}^{\prime}\right|^{2} \\
& +(3-2 \sqrt{2})\left|\boldsymbol{w}^{\prime}\right|^{2}+2(\sqrt{2}-1) \boldsymbol{u}_{1}^{\prime} \cdot \boldsymbol{w}^{\prime} \\
& -\sqrt{2}(\sqrt{2}-1)\left(\boldsymbol{v}_{1}^{\prime} \cdot \boldsymbol{w}^{\prime}+\boldsymbol{v}_{2}^{\prime} \cdot \boldsymbol{w}^{\prime}\right) \\
& -\sqrt{2}\left(\boldsymbol{u}_{1}^{\prime} \cdot \boldsymbol{v}_{1}^{\prime}+\boldsymbol{u}_{1}^{\prime} \cdot \boldsymbol{v}_{2}^{\prime}\right) \\
& -2 \sqrt{2}(\sqrt{2}-1)\left(\boldsymbol{u}_{2}^{\prime} \cdot \boldsymbol{v}_{1}^{\prime}-\boldsymbol{u}_{2}^{\prime} \cdot \boldsymbol{v}_{2}^{\prime}\right) \\
= & (18-10 \sqrt{2})+2(\sqrt{2}-1) x_{\mathrm{XA}_{1}} \\
& -\sqrt{2}(\sqrt{2}-1)\left(x_{\mathrm{XB}_{1}}+x_{\mathrm{XB}_{2}}\right) \\
& -\sqrt{2}\left(x_{\mathrm{A}_{1} \mathrm{~B}_{1}}+x_{\mathrm{A}_{1} \mathrm{~B}_{2}}\right) \\
& -2 \sqrt{2}(\sqrt{2}-1)\left(x_{\mathrm{A}_{2} \mathrm{~B}_{1}}-x_{\mathrm{A}_{2} \mathrm{~B}_{2}}\right) \\
= & 14-10 \sqrt{2}<0,
\end{aligned}
$$

which gives contradiction.

For larger values of $m$ and $n$, we extend the vector $\boldsymbol{x}$ by assigning zeros to additional coordinates. Then the new vector $\boldsymbol{x} \in \mathbb{R}^{\nabla E_{m, n}}$ lies in $\pi^{-1}\left(\mathcal{E}\left(\mathrm{K}_{m, n}\right)\right) \cap \operatorname{RMet}\left(\nabla \mathrm{K}_{m, n}\right)$ but not in $\mathcal{E}\left(\nabla \mathrm{K}_{m, n}\right)$.

We note several facts about the relation between this vector $\boldsymbol{x}$ and the Clauser-Horne-Shimony-Holt (CHSH) inequality $f_{\mathrm{CHSH}} \leq 2$ [7], where

$$
f_{\mathrm{CHSH}}=x_{\mathrm{A}_{1} \mathrm{~B}_{1}}+x_{\mathrm{A}_{1} \mathrm{~B}_{2}}+x_{\mathrm{A}_{2} \mathrm{~B}_{1}}-x_{\mathrm{A}_{2} \mathrm{~B}_{2}} .
$$

First, if we replace $x_{\mathrm{XB}_{1}}$ and $x_{\mathrm{XB}_{2}}$ by zero, then it becomes realizable in a quantum experiment. This is the famous example which violates the CHSH inequality maximally (the maximality comes from Tsirelson's theorem). Second, the vector $\boldsymbol{x}$ also maximizes $f_{\mathrm{CHSH}}$ in $\pi^{-1}\left(\mathcal{E}\left(\mathrm{K}_{2,2}\right)\right) \cap \operatorname{RMet}\left(\nabla \mathrm{K}_{2,2}\right)$, which is obvious from the fact that $f_{\mathrm{CHSH}}$ does not depend on any of $x_{\mathrm{XA}_{i}}$ or $x_{\mathrm{XB}_{j}}$. Third, the vector $\boldsymbol{x}$ maximizes $f=f_{\mathrm{CHSH}}-x_{\mathrm{XA}_{1}}+x_{\mathrm{XB}_{1}}+x_{\mathrm{XB}_{2}}$ in $\pi^{-1}\left(\mathcal{E}\left(\mathrm{K}_{2,2}\right)\right) \cap$ $\operatorname{RMet}\left(\nabla \mathrm{K}_{2,2}\right)$, attaining $2+\sqrt{2} \approx 3.4142$. On the other hand, the maximum of $f$ in $\mathcal{E}\left(\nabla \mathrm{K}_{2,2}\right) \cap \operatorname{RMet}\left(\nabla \mathrm{K}_{2,2}\right)$ is $9-4 \sqrt{2} \approx 3.3431$, achieved by

$$
\begin{gathered}
x_{\mathrm{XA}_{1}}^{\prime}=3-2 \sqrt{2}, x_{\mathrm{XA}_{2}}^{\prime}=0, x_{\mathrm{XB}_{1}}^{\prime}=x_{\mathrm{XB}_{2}}^{\prime}=\sqrt{2}-1, \\
x_{\mathrm{A}_{1} \mathrm{~B}_{1}}^{\prime}=x_{\mathrm{A}_{1} \mathrm{~B}_{2}}^{\prime}=5-3 \sqrt{2}, \\
x_{\mathrm{A}_{2} \mathrm{~B}_{1}}^{\prime}=2-\sqrt{2}, x_{\mathrm{A}_{2} \mathrm{~B}_{2}}^{\prime}=-2+\sqrt{2} .
\end{gathered}
$$

Actually, the proof above of Theorem 1 was obtained by solving the optimization problem to maximize $f$ in $\mathcal{E}\left(\nabla \mathrm{K}_{2,2}\right) \cap$ $\operatorname{RMet}\left(\nabla \mathrm{K}_{2,2}\right)$, which is a semidefinite program, by using SDPA (version 6.2.1) [8], and analyzing its dual optimal solution.

\section{CONCLUDING REMARKS}

We showed that the expected values of the single observables $A_{i}$ and $B_{j}$ can be used to improve the bound of the quantum correlation set implied by Tsirelson's theorem and the nonnegativity of the probabilities when the two parties have an arbitrary number of \pm 1 -valued observables.

Two implications of this are as follows. Firstly, Tsirelson's theorem gives a test for quantum theory itself: if pairwise correlations are found in nature that are not permitted by his theorem, then quantum theory is incomplete. Our result gives a stronger test, since correlations are possible that satisfy Tsirelson's theorem but are not permitted by quantum theory. For example, if the correlation used in the proof is realizable in nature, the quantum theory will be proven to be incomplete. Secondly, Tsirelson's theorem allows the efficient computation of the maximum violation of Bell inequalities, which have many applications in quantum communication and complexity [2], [9]. Our result allows for the computation of tighter bounds for inequalities involving the means of individual observables, as well as the observed pairwise correlations. For example, Collins and Gisin [10] show that the $I_{3322}$ Bell inequality $f_{3322} \leq 4$, where

$$
\begin{aligned}
f_{3322}= & -x_{\mathrm{XA}_{1}}-x_{\mathrm{XA}_{2}}+x_{\mathrm{XB}_{1}}+x_{\mathrm{XB}_{2}} \\
& +x_{\mathrm{A}_{1} \mathrm{~B}_{1}}+x_{\mathrm{A}_{1} \mathrm{~B}_{2}}+x_{\mathrm{A}_{1} \mathrm{~B}_{3}} \\
& +x_{\mathrm{A}_{2} \mathrm{~B}_{1}}+x_{\mathrm{A}_{2} \mathrm{~B}_{2}}-x_{\mathrm{A}_{2} \mathrm{~B}_{3}} \\
& +x_{\mathrm{A}_{3} \mathrm{~B}_{1}}-x_{\mathrm{A}_{3} \mathrm{~B}_{2}},
\end{aligned}
$$

and one can achieve $f_{3322}=5$ in $\mathcal{Q}_{\text {Cut }}(3,3)$. On the other hand, the inclusion (1) gives an upper bound $f_{3322} \leq 8(\sqrt{3}-$ $1) \approx 5.8564$ in $\mathcal{Q}_{\text {Cut }}(3,3)$. The inclusion (2) gives a tighter upper bound $f_{3322} \leq 2(\sqrt{3}+1) \approx 5.4641$ in $\mathcal{Q}_{\text {Cut }}(3,3)$.

This result motivates a search for a more ambitious example: the pairwise correlation functions are realizable in a classical 
correlation experiment, but if we take the mean values into account, the entire result is not realizable even in quantum correlation experiments. In this direction, we have the following result, whose proof will be available in near future.

Theorem 2: (i) For $m=n=3$, there exists a vector $\boldsymbol{x} \in \mathbb{R}^{\nabla E_{m, n}}$ which is no-signalling but not realizable in quantum correlation experiments, such that if mean values of individual observables are discarded, the pairwise correlation functions are realizable in a classical correlation experiment. An example of such a vector is as follows:

$$
\begin{gathered}
x_{\mathrm{XA}_{1}}=x_{\mathrm{XA}_{2}}=x_{\mathrm{XA}_{3}}=x_{\mathrm{XB}_{1}}=x_{\mathrm{XB}_{2}}=x_{\mathrm{XB}_{3}}=1 / 3, \\
x_{\mathrm{A}_{1} \mathrm{~B}_{1}}=x_{\mathrm{A}_{2} \mathrm{~B}_{2}}=1, \\
x_{\mathrm{A}_{1} \mathrm{~B}_{2}}=x_{\mathrm{A}_{1} \mathrm{~B}_{3}}=x_{\mathrm{A}_{2} \mathrm{~B}_{1}}=x_{\mathrm{A}_{2} \mathrm{~B}_{3}} \\
=x_{\mathrm{A}_{3} \mathrm{~B}_{1}}=x_{\mathrm{A}_{3} \mathrm{~B}_{2}}=x_{\mathrm{A}_{3} \mathrm{~B}_{3}}=-1 / 3 .
\end{gathered}
$$

(ii) There is no such vector if $\min \{m, n\}=2$.

Now that we know the inclusion (3) is proper, the next question is: how much is the difference between the two sides of the inequality (3)? Another, more challenging, problem is whether equality holds in the inequality (2).

\section{ACKNOWLEDGMENT}

We would like to thank Hiroshi Imai for valuable comments and helpful discussions. The first author is supported by N.S.E.R.C. and F.Q.R.N.T research grants, and the second author is supported by the Japan Society for the Promotion of Science Fellowship and the Grant-In-Aid for JSPS Fellows No. 17-50212.

\section{REFERENCES}

[1] B. S. Tsirelson, "Some results and problems on quantum Bell-type inequalities," Hadronic Journal Supplement, vol. 8, no. 4, pp. 329-345, 1993.

[2] R. F. Werner and M. M. Wolf, "Bell inequalities and entanglement," Quantum Information and Computation, vol. 1, no. 3, pp. 1-25, Oct. 2001, arXiv:quant-ph/0107093.

[3] B. S. Cirel'son, "Quantum generalizations of Bell's inequality," Letters in Mathematical Physics, vol. 4, no. 2, pp. 93-100, 1980.

[4] B. S. Tsirel'son, "Quantum analogues of the Bell inequalities: The case of two spatially separated domains," Journal of Soviet Mathematics, vol. 36, pp. 557-570, 1987.

[5] M. M. Deza and M. Laurent, Geometry of Cuts and Metrics, ser Algorithms and Combinatorics. Springer, May 1997, vol. 15.

[6] D. Avis, H. Imai, and T. Ito, "On the relationship between convex bodies related to correlation experiments with dichotomic observables," Journal of Physics A: Mathematical and General, vol. 39, no. 36, pp. $11283-$ 11299 , Sept. 2006, arXiv:quant-ph/0605148.

[7] J. F. Clauser, M. A. Horne, A. Shimony, and R. A. Holt, "Proposed experiment to test local hidden-variable theories," Physical Review Letters, vol. 23, no. 15, pp. 880-884, Oct. 1969.

[8] K. Fujisawa, M. Kojima, K. Nakata, and M. Yamashita, "SDPA (SemiDefinite Programming Algorithm) user's manual: Version 6.2.0," Dept. Math. \& Comp. Sciences, Tokyo Institute of Technology, Research Report B-308, Dec. 1995, revised to version 6.2.1 in Sept. 2005, See also http://grid.r.dendai.ac.jp/sdpa/.

[9] R. Cleve, P. Høyer, B. Toner, and J. Watrous, "Consequences and limits of nonlocal strategies," in Proceedings of 19th IEEE Annual Conference on Computational Complexity (CCC'04), June 2004, pp. 236-249, arXiv:quant-ph/0404076.

[10] D. Collins and N. Gisin, "A relevant two qubit Bell inequality inequivalent to the CHSH inequality," Journal of Physics A: Mathematical and General, vol. 37, no. 5, pp. 1775-1787, Feb. 2004, arXiv:quant$\mathrm{ph} / 0306129$. 